\title{
Bidirectional Relay Communication with Additional Private Message
}

\author{
Konstantinos Benidis, Rafael F. Schaefer, and Holger Boche \\ Lehrstuhl für Theoretische Informationstechnik \\ Technische Universität München, Germany
}

\begin{abstract}
The spectral efficiency of wireless networks can be significantly improved by wisely integrating multiple services at the physical layer. Here, we consider physical layer service integration in bidirectional relay networks, in which a relay node establishes a bidirectional communication between two other nodes using a decode-and-forward protocol. In the broadcast phase the relay efficiently integrates an additional private message for one node which requires the study of the bidirectional broadcast channel with additional private message. The corresponding capacity regions for discrete memoryless and MIMO Gaussian channels are derived.
\end{abstract}

\section{INTRODUCTION}

Recently, several key techniques have been identified which have the potential to improve the performance of next generation wireless networks like the use of multiple transmit and receive antennas (MIMO) [1]. The efficient implementation of multiple services at the physical layer has been identified as a promising research direction. In current cellular systems, operators already offer not only traditional services such as (bidirectional) voice communication, but also further private services intended for specific users. This is realized by policies that allocate different services on different logical channels, which is quite inefficient in general. Thus, physical layer service integration has the potential to significantly increase the spectral efficiency of future wireless networks.

Another key technique is the concept of bidirectional relaying which has the advantage of exploiting the property of bidirectional communication to reduce the inherent loss in spectral efficiency induced by half-duplex relays. Bidirectional relaying applies to three-node networks where a relay node establishes bidirectional communication between two other nodes using a decode-and-forward protocol. In the initial multiple access (MAC) phase two nodes transmit their messages to the relay node which decodes them. Then, in the bidirectional broadcast (BBC) phase the relay re-encodes and transmits both messages in a way that both receiving nodes can decode their intended message, using their own message as side information.

Bidirectional relaying and its extensions are widely studied. Capacity achieving strategies can be found in [2-4] for discrete memoryless channels and in [5] for MIMO Gaussian channels. Based on these results, optimal transmit strategies are then

The work was supported by the German Research Foundation (DFG) under Grant BO 1734/25-1.
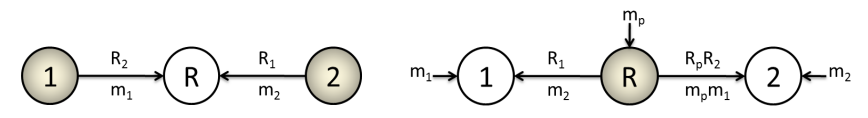

(a) MAC phase

(b) BBC phase

Fig. 1. Physical layer service integration in bidirectional relay networks. In the initial MAC phase, nodes 1 and 2 transmit their messages $m_{1}$ and $m_{2}$ with rates $R_{2}$ and $R_{1}$ to the relay node. Then, in the $\mathrm{BBC}$ phase, the relay forwards the messages $m_{1}$ and $m_{2}$ and adds a private message $m_{p}$ with rate $R_{p}$ to the communication for node 2 .

analyzed in $[6,7]$. The efficient integration of bidirectional relaying in a cellular downlink is discussed in [8]. Physical layer service integration with secrecy constraints is analyzed in [9]. The scenario where the relay serves multiple pairs of users is addressed for instance in [10-12]. The problem of channel estimation for bidirectional relaying is studied in [13].

In this paper, we consider physical layer service integration in bidirectional relay networks. Besides the transmission of the bidirectional messages, the relay integrates an additional private message as shown in Figure 1. Therefore, we introduce the bidirectional broadcast channel $(B B C)$ with additional private message in Section II. The receiving nodes can use their own messages from the previous phase for decoding, so that this differs from the classical broadcast scenario. The corresponding capacity regions for DMC and MIMO Gaussian channels are subsequently derived in Sections II and III. Then in Section IV, we discuss the problem of finding the optimal transmit covariance matrices, which is a non-trivial task as the corresponding optimization problems are in general nonconvex. Finally, we conclude the paper in Section V. ${ }^{1}$

\section{BidiRECTIONAL BROADCAST CHANNEL WITH Additional Private Message}

In this section we analyze the integration of an additional private message for discrete memoryless channels.

${ }^{1}$ Notation: Vectors and matrices are denoted by bold lower case letters and bold capital letters; random variables are denoted by non-italic capital letters and their realizations and ranges by lower case italic letters and script letters, respectively; $I(\cdot ; \cdot)$ is the mutual information; $X-Y-Z$ denotes a Markov chain of the random variables $X, Y$, and $Z$ in this order; $\mathbb{N}$ and $\mathbb{R}_{+}$are the sets of non-negative integers and non-negative real numbers; $(\cdot)^{T}$ idenotes the transpose; $\operatorname{tr}(\cdot)$ and $\operatorname{det}(\cdot)$ are the trace and the determinant of a matrix; $\boldsymbol{A} \succeq \boldsymbol{B}$ means the matrix $\boldsymbol{A}-\boldsymbol{B}$ is positive semidefinite; $A_{\epsilon}^{(n)}(\cdot)$ is the set of (weakly) typical sequences, cf. for example [14]. 


\section{A. Physical Layer Description and Capacity Result}

Let $\mathcal{X}$ and $\mathcal{Y}_{k}, k=1,2$, be finite input and output sets. A discrete memoryless broadcast channel is defined by a family $\left\{W^{(n)}: \mathcal{X}^{n} \rightarrow \mathcal{Y}_{1}^{n} \times \mathcal{Y}_{2}^{n}\right\}_{n \in \mathbb{N}}$ of probability transition functions given by $W^{(n)}\left(y_{1}^{n}, y_{2}^{n} \mid x^{n}\right):=\prod_{i=1}^{n} W\left(y_{1, i}, y_{2, i} \mid x_{i}\right)$ for a probability transition function $W: \mathcal{X} \rightarrow \mathcal{Y}_{1} \times \mathcal{Y}_{2}$. We do not allow any cooperation between the receiving nodes so it is sufficient to consider the marginal conditional probabilities $W_{k}^{(n)}:=\prod_{i=1}^{n} W\left(y_{k, i} \mid x_{i}\right)$ for $k=1,2$.

We now consider a block code of arbitrary but fixed length $n$. The set of bidirectional messages of node $k, k=1,2$ is denoted by $\mathcal{M}_{k}:=\left\{1, \ldots, M_{k}^{(n)}\right\}$ which is also known at the relay node. Furthermore, the set of the additional private messages of the relay node is denoted by $\mathcal{M}_{p}:=\left\{1, \ldots, M_{p}^{(n)}\right\}$. We introduce the abbreviation $\mathcal{M}:=\mathcal{M}_{p} \times \mathcal{M}_{1} \times \mathcal{M}_{2}$.

In the BBC phase, we assume that the relay has successfully decoded both bidirectional messages $m_{1} \in \mathcal{M}_{1}$ and $m_{2} \in \mathcal{M}_{2}$ which have been transmitted in the MAC phase by nodes 1 and 2. Besides these two private messages, the relay integrates an additional private message $m_{p} \in \mathcal{M}_{p}$ for node 2 .

Definition 1: An $\left(M_{p}^{(n)}, M_{1}^{(n)}, M_{2}^{(n)}, n\right)$-code for the BBC with additional private message consists of one encoder at the relay node

$$
f: \mathcal{M}_{p} \times \mathcal{M}_{1} \times \mathcal{M}_{2} \rightarrow \mathcal{X}^{n}
$$

and decoders at nodes 1 and 2

$$
\begin{aligned}
& g_{1}: \mathcal{Y}_{1}^{n} \times \mathcal{M}_{1} \rightarrow \mathcal{M}_{2} \\
& g_{2}: \mathcal{Y}_{2}^{n} \times \mathcal{M}_{2} \rightarrow \mathcal{M}_{p} \times \mathcal{M}_{1} .
\end{aligned}
$$

Now, we assume that the relay has sent the message $m=\left(m_{p}, m_{1}, m_{2}\right)$ and nodes 1 and 2 have received $y_{1}^{n}$ and $y_{2}^{n}$, respectively. Then, the decoder at node 1 is in error if $g_{1}\left(y_{1}^{n}, m_{1}\right) \neq m_{2}$. Accordingly, the decoder at node 2 is in error if $g_{2}\left(y_{2}^{n}, m_{2}\right) \neq\left(m_{p}, m_{1}\right)$. This allows us to introduce the notation for the average probability of error for the $k$ th node

$$
\mu_{k}^{(n)}=\frac{1}{|\mathcal{M}|} \sum_{m \in \mathcal{M}} \lambda_{k}(m)
$$

with $\lambda_{1}(m)=\mathbb{P}\left\{g_{1}\left(y_{1}^{n}, m_{1}\right) \neq m_{2} \mid m\right.$ was sent $\}$ and $\lambda_{2}(m)=\mathbb{P}\left\{g_{2}\left(y_{2}^{n}, m_{2}\right) \neq\left(m_{p}, m_{1}\right) \mid m\right.$ was sent $\}$.

Definition 2: A rate triple $\left(R_{p}, R_{1}, R_{2}\right) \in \mathbb{R}_{+}^{3}$ is said to be achievable for the $\mathrm{BBC}$ with additional private message if for any $\delta>0$ there exists an $n(\delta) \in \mathbb{N}$ and a sequence of $\left(M_{p}^{(n)}, M_{1}^{(n)}, M_{2}^{(n)}, n\right)$-codes such that for all $n \geq n(\delta)$ we have $\frac{1}{n} \log \left|\mathcal{M}_{p}\right| \geq R_{p}-\delta, \frac{1}{n} \log \left|\mathcal{M}_{2}\right| \geq R_{1}-\delta$ and $\frac{1}{n} \log \left|\mathcal{M}_{1}\right| \geq R_{2}-\delta$ while $\mu_{1}^{(n)}, \mu_{2}^{(n)} \rightarrow 0$ as $n \rightarrow \infty$. The set of all achievable rate triples is the capacity region of the $\mathrm{BBC}$ with additional private message and is denoted by $\mathcal{C}_{\mathrm{BBC}}$.

Theorem 1: The capacity region $\mathcal{C}_{\mathrm{BBC}}$ of the discrete memoryless $\mathrm{BBC}$ with additional private message is the set of all rate triples $\left(R_{p}, R_{1}, R_{2}\right) \in \mathbb{R}_{+}^{3}$ that satisfy:

$$
\begin{aligned}
R_{1} & \leq I\left(U ; Y_{1}\right) \\
R_{p} & \leq I\left(X ; Y_{2} \mid U\right) \\
R_{p}+R_{2} & \leq I\left(X ; Y_{2}\right)
\end{aligned}
$$

for random variables $U-X-\left(Y_{1}, Y_{2}\right)$. The cardinality of the range of $U$ can be bounded by $|\mathcal{U}| \leq \min \left\{|\mathcal{X}|,\left|\mathcal{Y}_{1}\right|,\left|\mathcal{Y}_{2}\right|\right\}+1$.

Remark 1: Applying some reformulations and substitutions the result can also be deduced from [4], which studies a similar broadcast scenario, where the receivers have (partial) side information about the transmitted messages available.

Theorem 1 is proved in the following two subsections.

\section{B. Proof of Achievability}

1) Random Codebook Generation: For any $\delta>0$ we define the message sets $\mathcal{M}_{p}, \mathcal{M}_{1}, \mathcal{M}_{2}$ such that $\left|\mathcal{M}_{p}\right|=$ $\left\lfloor 2^{n\left(R_{p}-\frac{\delta}{2}\right)}\right\rfloor,\left|\mathcal{M}_{1}\right|=\left\lfloor 2^{n\left(R_{2}-\frac{\delta}{2}\right)}\right\rfloor$ and $\left|\mathcal{M}_{2}\right|=\left\lfloor 2^{n\left(R_{1}-\frac{\delta}{2}\right)}\right\rfloor$. Randomly and independently we generate $\left|\mathcal{M}_{1}\right|\left|\mathcal{M}_{2}\right|$ codewords ("cloud centers") $u^{n}\left(m_{1}, m_{2}\right)$, each according to $\prod_{i=1}^{n} p_{U}\left(u_{i}\right)$. For each codeword $u^{n}\left(m_{1}, m_{2}\right)$ randomly and conditionally independently we generate $\left|\mathcal{M}_{p}\right|$ codewords ("satellites") $x^{n}\left(m_{p}, m_{1}, m_{2}\right)$, each according to $\prod_{i=1}^{n} p_{X \mid U}\left(x_{i} \mid u_{i}\right)$.

2) Encoding: To send the message $\left(m_{p}, m_{1}, m_{2}\right)$ the relay transmits the codeword $x^{n}\left(m_{p}, m_{1}, m_{2}\right)$.

3) Decoding: The receiving nodes use typical set decoding. The decoder in node 1 decodes the "cloud center" $u^{n}\left(m_{1}, m_{2}\right)$. It declares that a message $\hat{m}_{2}$ was sent if it is the unique message such that $\left(u^{n}\left(m_{1}, \hat{m}_{2}\right), y_{1}^{n}\right) \in$ $A_{\epsilon}^{(n)}\left(U Y_{1}\right)$. Respectively, the decoder in node 2 decodes the "satellite" $x^{n}\left(m_{p}, m_{1}, m_{2}\right)$ and it declares that the messages $\left(\hat{m}_{p}, \hat{m}_{1}\right)$ were sent if they are the unique messages such that $\left(u^{n}\left(\hat{m}_{1}, m_{2}\right), x^{n}\left(\hat{m}_{p}, \hat{m}_{1}, m_{2}\right), y_{2}^{n}\right) \in A_{\epsilon}^{(n)}\left(U X Y_{2}\right)$.

3) Analysis of the Probability of Error: For the decoder in node 1 , we define the error events

$$
\begin{aligned}
& E_{11}:=\left\{\left(u^{n}, y_{1}^{n}\right) \notin A_{\epsilon}^{(n)}\left(U Y_{1}\right)\right\} \\
& E_{12}:=\left\{\exists m_{2}^{\prime} \neq m_{2}:\left(u^{n}, y_{1}^{n}\right) \in A_{\epsilon}^{(n)}\left(U Y_{1}\right)\right\} .
\end{aligned}
$$

From the union bound we have

$$
\mu_{1}^{(n)} \leq \mathbb{P}\left\{E_{11}\right\}+\mathbb{P}\left\{E_{12}\right\} .
$$

From the Law of Large Numbers we know that $\mathbb{P}\left\{E_{11}\right\} \rightarrow 0$ as $n \rightarrow \infty$. For the second error event, with $\delta=10 \epsilon$, by using standard arguments we can show that

$$
\mathbb{P}\left\{E_{12}\right\} \leq 2^{-n\left(I\left(U ; Y_{1}\right)-R_{1}+2 \epsilon\right)}
$$

For the decoder in node 2, we define the error events

$$
\begin{aligned}
& E_{21}:=\left\{\left(u^{n}, x^{n}, y_{2}^{n}\right) \notin A_{\epsilon}^{(n)}\left(U X Y_{2}\right)\right\} \\
& E_{22}:=\left\{\exists m_{1}^{\prime} \neq m_{1}:\left(u^{n}, x^{n}, y_{2}^{n}\right) \in A_{\epsilon}^{(n)}\left(U X Y_{2}\right)\right\} \\
& E_{23}:=\left\{\exists m_{p}^{\prime} \neq m_{p}:\left(u^{n}, x^{n}, y_{2}^{n}\right) \in A_{\epsilon}^{(n)}\left(U X Y_{2}\right)\right\} \\
& E_{24}:=\left\{\exists\left(m_{p}^{\prime}, m_{1}^{\prime}\right) \neq\left(m_{p}, m_{1}\right):\left(u^{n}, x^{n}, y_{2}^{n}\right) \in A_{\epsilon}^{(n)}\left(U X Y_{2}\right)\right\} .
\end{aligned}
$$

From the union bound we have $\mu_{2}^{(n)} \leq \mathbb{P}\left\{E_{21}\right\}+\mathbb{P}\left\{E_{22}\right\}+$ $\mathbb{P}\left\{E_{23}\right\}+\mathbb{P}\left\{E_{24}\right\}$. 
Again, from the Law of Large Numbers we have $\mathbb{P}\left\{E_{21}\right\} \rightarrow$ 0 as $n \rightarrow \infty$ and similarly we can show that

$$
\begin{aligned}
& \mathbb{P}\left\{E_{22}\right\} \leq 2^{-n\left(I\left(U, X ; Y_{2}\right)-R_{2}+2 \epsilon\right)} \\
& \mathbb{P}\left\{E_{23}\right\} \leq 2^{-n\left(I\left(X ; Y_{2} \mid U\right)-R_{p}+\epsilon\right)} \\
& \mathbb{P}\left\{E_{24}\right\} \leq 2^{-n\left(I\left(U, X ; Y_{2}\right)-R_{p}-R_{2}+2 \epsilon\right)} .
\end{aligned}
$$

Now, by arguing that the average probabilities of error should be arbitrarily close to zero and by observing that $U-X-\left(Y_{1}, Y_{2}\right)$, we get the capacity region of Theorem 1 . The cardinality bound of the random variable $U$, i.e. $|\mathcal{U}| \leq$ $\min \left\{|\mathcal{X}|,\left|\mathcal{Y}_{1}\right|,\left|\mathcal{Y}_{2}\right|\right\}+1$, follows from [15, Appendix C] and [16]. This completes the proof of achievability.

\section{Proof of Converse}

Trying to prove the converse directly for the expression (1) does not appear to be feasible. This is mainly because it is difficult to find an identification of the auxiliary random variable $U$ that works for the first two inequalities. As in [15], we introduce the equivalent region consisting of all rate triples $\left(R_{p}, R_{1}, R_{2}\right) \in \mathbb{R}_{+}^{3}$ such that:

$$
\begin{aligned}
R_{1} & \leq I\left(U ; Y_{1}\right) \\
R_{p}+R_{1} & \leq I\left(X ; Y_{2} \mid U\right)+I\left(U ; Y_{1}\right) \\
R_{p}+R_{2} & \leq I\left(X ; Y_{2}\right) .
\end{aligned}
$$

The proof of equivalence of the regions (1) and (3) involves rate splitting and the Fourier-Motzkin elimination procedure. We omit the details due to space constraints. Now, we can prove the converse for the equivalent region (3).

By Fano's inequality it is straightforward that

$$
\begin{aligned}
n R_{1} & \leq I\left(M_{1}, M_{2} ; Y_{1}^{n}\right)+n \epsilon_{1}^{(n)} \\
n\left(R_{p}+R_{1}\right) & \leq I\left(M_{p} ; Y_{2}^{n} \mid M_{1}, M_{2}\right)+I\left(M_{1}, M_{2} ; Y_{1}^{n}\right)+n \epsilon_{2}^{(n)} \\
n\left(R_{p}+R_{2}\right) & \leq I\left(M_{p}, M_{1}, M_{2} ; Y_{2}^{n}\right)+n \epsilon_{3}^{(n)} .
\end{aligned}
$$

By using the Csiszár sum identity [17, Lemma 7], [18], the mutual information chain rules and other standard arguments in the above inequalities, we can show that

$$
\begin{aligned}
R_{1} & \leq \frac{1}{n} \sum_{i=1}^{n} I\left(U_{i} ; Y_{1, i}\right)+\epsilon_{1}^{(n)} \\
R_{p}+R_{1} & \leq \frac{1}{n} \sum_{i=1}^{n}\left(I\left(X_{i} ; Y_{2, i} \mid U_{i}\right)+I\left(U_{i} ; Y_{1, i}\right)\right)+\epsilon_{2}^{(n)} \\
R_{p}+R_{2} & \leq \frac{1}{n} \sum_{i=1}^{n} I\left(X_{i} ; Y_{2, i}\right)+\epsilon_{3}^{(n)}
\end{aligned}
$$

where the auxiliary random variable identification is $U_{i}=$ $\left(M_{1}, M_{2}, Y_{2}^{i-1}, Y_{1, i+1}^{n}\right)$.

We can rewrite the inequalities (5a)-(5c) by introducing a "time-sharing" random variable $T$, where $T=i \in\{1,2, \ldots, n\}$ with probability $\frac{1}{n}$,

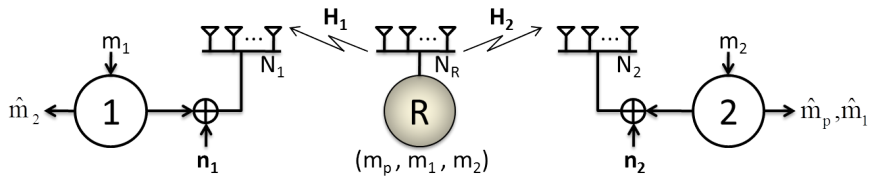

Fig. 2. MIMO Gaussian BBC with additional private message.

i.e., we have $p_{T U_{T} X_{T} Y_{1, T} Y_{2, T}}\left(i, u_{i}, x_{i}, y_{1, i}, y_{2, i}\right)=$ $\frac{1}{n} p_{U_{i} X_{i}}\left(u_{i}, x_{i}\right) p_{Y_{1} Y_{2} \mid X}\left(y_{1}, y_{2} \mid x\right)$. For (5a) we have

$$
R_{1} \leq \sum_{i=1}^{n} \mathbb{P}(T=i) I\left(U_{i} ; Y_{1, i}\right)+\epsilon_{1}^{(n)}=I\left(U_{T} ; Y_{1, T}\right)+\epsilon_{1}^{(n)}
$$

where the distributions of the new random variables $U_{T}, X_{T}, Y_{1, T}, Y_{2, T}$ depends on $T$ in the same way as the distributions of $U_{i}, X_{i}, Y_{1, i}, Y_{2, i}$ depend on $i$. We define $U:=U_{T}, X:=X_{T}, Y_{1}:=Y_{1, T}, Y_{2}:=Y_{2, T}$. Now we can bound (5a) as $R_{1} \leq I\left(U ; Y_{1}\right)+\epsilon_{1}^{(n)}$. Similarly we can bound (5b) and (5c) as $R_{p}+R_{1} \leq I\left(X ; Y_{2} \mid U\right)+I\left(U ; Y_{1}\right)+\epsilon_{2}^{(n)}$ and $R_{p}+R_{2} \leq I\left(X ; Y_{2}\right)+\epsilon_{3}^{(n)}$, where $\epsilon_{k}^{(n)} \rightarrow 0, k=1,2,3$, as $n \rightarrow \infty$. This completes the proof of converse.

\section{MIMO GAUSSIAN CHANNEL}

In this section we consider physical layer service integration in mulitantenna bidirectional relay networks and prove the corresponding capacity region of the MIMO Gaussian BBC with additional private message.

\section{A. Physical Layer Description and Capacity Result}

Let $N_{R}$ be the number of antennas at the relay node and $N_{k}$ be the number of antennas at node $k, k=1,2$ as shown in Figure 2. The discrete-time real-valued input-output relation between the relay node and node $k, k=1,2$, can now be modeled as

$$
\boldsymbol{y}_{k}=\boldsymbol{H}_{k} \boldsymbol{x}+\boldsymbol{n}_{k}
$$

where $\boldsymbol{y}_{k} \in \mathbb{R}^{N_{k} \times 1}$ denotes the output at node $k, \boldsymbol{H}_{k} \in$ $\mathbb{R}^{N_{k} \times N_{R}}$ is the multiplicative channel matrix, $\boldsymbol{x} \in \mathbb{R}^{N_{R} \times 1}$ is the input of the relay node and $\boldsymbol{n}_{k} \in \mathbb{R}^{N_{k} \times 1}$ is independent additive noise according to a circular symmetric Gaussian distribution $\mathcal{N}\left(\mathbf{0}, \sigma_{k}^{2} \boldsymbol{I}_{N_{k}}\right)$.

As in $[9,19,20]$, we consider two different kinds of power constraints: a total power constraint and a covariance constraint. An input sequence $\boldsymbol{x}^{n}=\left(\boldsymbol{x}_{1}, \boldsymbol{x}_{2}, \ldots, \boldsymbol{x}_{n}\right)$ of length $n$ satisfies a total power constraint $P$ if

$$
\frac{1}{n} \sum_{i=1}^{n} \boldsymbol{x}_{i}^{T} \boldsymbol{x}_{i} \leq P .
$$

Similarly $\boldsymbol{x}^{n}$ satisfies the covariance constraint $\boldsymbol{S}$ if $\frac{1}{n} \sum_{i=1}^{n} \boldsymbol{x}_{i} \boldsymbol{x}_{i}^{T} \preceq \boldsymbol{S}$ where $\boldsymbol{S} \succeq \mathbf{0}$ is a positive semidefinite matrix.

Theorem 2: The capacity region $\mathcal{C}_{\mathrm{BBC}}^{\mathrm{MIMO}}(\boldsymbol{S})$ of the MIMO Gaussian BBC with additional private message under the covariance constraint $\boldsymbol{S}$ is:

$$
\mathcal{C}_{\mathrm{BBC}}^{\mathrm{MIMO}}(\boldsymbol{S})=\bigcup_{\mathbf{0} \preceq \boldsymbol{Q}_{p} \preceq \boldsymbol{S}} \mathcal{R}\left(\boldsymbol{S}, \boldsymbol{Q}_{p}\right)
$$


where $\mathcal{R}\left(\boldsymbol{S}, \boldsymbol{Q}_{p}\right)$ is the set of all rate triples $\left(R_{p}, R_{1}, R_{2}\right) \in$ $\mathbb{R}_{+}^{3}$ that satisfy:

$$
\begin{aligned}
R_{1} & \leq \frac{1}{2} \log \operatorname{det}\left(\frac{\boldsymbol{H}_{1} \boldsymbol{S} \boldsymbol{H}_{1}^{T}+\sigma_{1}^{2} \boldsymbol{I}_{N_{1}}}{\boldsymbol{H}_{1} \boldsymbol{Q}_{p} \boldsymbol{H}_{1}^{T}+\sigma_{1}^{2} \boldsymbol{I}_{N_{1}}}\right) \\
R_{p} & \leq \frac{1}{2} \log \operatorname{det}\left(\boldsymbol{I}_{N_{2}}+\frac{1}{\sigma_{2}^{2}} \boldsymbol{H}_{2} \boldsymbol{Q}_{p} \boldsymbol{H}_{2}^{T}\right) \\
R_{p}+R_{2} & \leq \frac{1}{2} \log \operatorname{det}\left(\boldsymbol{I}_{N_{2}}+\frac{1}{\sigma_{2}^{2}} \boldsymbol{H}_{2} \boldsymbol{S} \boldsymbol{H}_{2}^{T}\right) .
\end{aligned}
$$

The capacity region under the total power constraint can be derived from the following corollary [20, Lemma 1].

Corollary 1: The extension of Theorem 2 to the total power constraint is immediate and given by

$$
C_{\mathrm{BBC}}^{\mathrm{MIMO}}(P)=\bigcup_{\operatorname{tr}(\boldsymbol{S}) \preceq P} C_{\mathrm{BBC}}^{\mathrm{MIMO}}(\boldsymbol{S}) .
$$

Theorem 2 is proved in the following two subsections.

\section{B. Proof of Achievability}

To obtain the desired region (7) we follow the proof of the discrete case, cf. Section II, with a proper choice of auxiliary and input random variables. More precisely, with $\boldsymbol{U} \sim \mathcal{N}\left(\mathbf{0}, \boldsymbol{S}-\boldsymbol{Q}_{p}\right)$ for the bidirectional messages, $\boldsymbol{V} \sim$ $\mathcal{N}\left(\mathbf{0}, \boldsymbol{Q}_{p}\right)$ for the additional private message, and further $\boldsymbol{X}=\boldsymbol{U}+\boldsymbol{V} \sim \mathcal{N}(\mathbf{0}, \boldsymbol{S})$, the region (7) follows immediately from Theorem 1. Therefore we omit the details for brevity.

\section{Proof of Converse}

To establish the converse it remains to show that no other rate triples than those characterized in (7) are achievable for some $\mathbf{0} \preceq \boldsymbol{Q}_{p} \preceq S$.

We define the single user capacity of the channel between the relay and user 2 as:

$$
\mathcal{C}_{2}=\frac{1}{2} \log \operatorname{det}\left(\boldsymbol{I}_{N_{2}}+\frac{1}{\sigma_{2}^{2}} \boldsymbol{H}_{2} \boldsymbol{S} \boldsymbol{H}_{2}^{T}\right) .
$$

As both the bidirectional message $m_{1}$ and the private message $m_{p}$ have to be successfully received by user 2 , we must have

$$
R_{p}+R_{2} \leq \mathcal{C}_{2}=\frac{1}{2} \log \operatorname{det}\left(\boldsymbol{I}_{N_{2}}+\frac{1}{\sigma_{2}^{2}} \boldsymbol{H}_{2} \boldsymbol{S} \boldsymbol{H}_{2}^{T}\right)
$$

which proves the inequality $(7 \mathrm{c})$.

Proving the inequalities (7a) and (7b) is not a trivial problem. Therefore, we use existing bounds of a similar scenario and with proper arguments we conclude to the desired result.

It is proved in [20] that the capacity region of a multiantenna Gaussian broadcast channel with one private message at rate $R_{p}^{\prime}$, and one common message at rate $R_{c}$ is the rate pair

$$
\begin{aligned}
R_{p}^{\prime} & \leq \frac{1}{2} \log \operatorname{det}\left(\boldsymbol{I}_{N_{1}}+\frac{1}{\sigma_{1}^{2}} \boldsymbol{H}_{1} \boldsymbol{Q}_{p}^{\prime} \boldsymbol{H}_{1}^{T}\right) \\
R_{c} & \leq \min \left(R_{c}^{1}, R_{c}^{2}\right)
\end{aligned}
$$

where

$$
R_{c}^{k}=\frac{1}{2} \log \operatorname{det}\left(\frac{\boldsymbol{H}_{k} \boldsymbol{S}^{\prime} \boldsymbol{H}_{k}^{T}+\sigma_{i}^{2} \boldsymbol{I}_{N_{k}}}{\boldsymbol{H}_{k} \boldsymbol{Q}_{p}^{\prime} \boldsymbol{H}_{k}^{T}+\sigma_{i}^{2} \boldsymbol{I}_{N_{k}}}\right)
$$

for some $\mathbf{0} \preceq Q_{p}^{\prime} \preceq S^{\prime}$.

We can interpret the united bidirectional message that contains both messages $m_{1}$ and $m_{2}$ (after the relay re-encoding), as a common message that is partially known from the two users. We set $R_{2}=0$ thus we "maximize" the bidirectional (common) rate $R_{1}$ of the message $m_{2}$. With this reformulation, our problem corresponds exactly to the similar scenario of one private and one common message. Now, we can claim that we are not interested in user 2 to be able to decode the message $m_{2}$ because there is already available as side information. So, we can discard the minimum from (9b) and keep only the corresponding rate for the channel of user 1 . With the above arguments we get an exact correspondance of the rate pairs $\left(R_{p}, R_{1}\right)$ and $\left(R_{p}^{\prime}, R_{c}^{1}\right)$ between the two scenarios. This gives us the desired bounds (7a) and (7b) and completes the proof of converse.

\section{DISCUSSION}

The optimal transmit covariance matrices are determined by non-convex optimization problems and so the weighted rate sum optimal rate triples as well. Hence, obtaining the boundary of the capacity region (6) is in general non-trivial. For the MISO scenario we can reformulate the optimization problem in such a way that it becomes convex and therewith tractable.

In the MISO case, the channel matrices $\boldsymbol{H}_{k}$ reduce to vectors $\boldsymbol{h}_{k}^{T}, k=1,2$. Here, we use the total power constraint $P$ instead of the covariance constraint $S$.

Similar to the MIMO case we assume that $U \sim \mathcal{N}\left(\mathbf{0}, Q_{12}\right)$, $\boldsymbol{V} \sim \mathcal{N}\left(\mathbf{0}, \boldsymbol{Q}_{p}\right)$ and $\boldsymbol{X}=\boldsymbol{U}+\boldsymbol{V} \sim \mathcal{N}\left(\mathbf{0}, \boldsymbol{Q}_{12}+\boldsymbol{Q}_{p}\right)$ where we require a total power constraint $\operatorname{tr}\left(\boldsymbol{Q}_{12}+\boldsymbol{Q}_{p}\right) \leq P$. Now, in an exact correspondance to region (7), we can write that $\mathcal{R}\left(\boldsymbol{Q}_{12}, \boldsymbol{Q}_{p}\right)$ is the set of all rate triples $\left(R_{p}, R_{1}, R_{2}\right) \in \mathbb{R}_{+}^{3}$ that satisfy

$$
\begin{aligned}
R_{1} & \leq \frac{1}{2} \log \left(1+\frac{\boldsymbol{h}_{1}^{T} \boldsymbol{Q}_{12} \boldsymbol{h}_{1}}{\boldsymbol{h}_{1}^{T} \boldsymbol{Q}_{p} \boldsymbol{h}_{1}+\sigma_{1}^{2}}\right) \\
R_{p} & \leq \frac{1}{2} \log \left(1+\frac{1}{\sigma_{2}^{2}} \boldsymbol{h}_{2}^{T} \boldsymbol{Q}_{p} \boldsymbol{h}_{2}\right) \\
R_{p}+R_{2} & \leq \frac{1}{2} \log \left(1+\frac{1}{\sigma_{2}^{2}} \boldsymbol{h}_{2}^{T}\left(\boldsymbol{Q}_{12}+\boldsymbol{Q}_{p}\right) \boldsymbol{h}_{2}\right) .
\end{aligned}
$$

Now we consider a slightly different problem. As in [20] and [9], instead of finding the best possible rates for a given power constraint, we find the minimum power required to achieve a set of minimal rate requirements. Furthermore, instead of considering the rates themselves, we can equivalently give minimum requirements on the SNIRs of the different users. With the above arguments we can write the following optimization problem which will allow us to calculate $\mathcal{R}\left(\boldsymbol{Q}_{12}, \boldsymbol{Q}_{p}\right)$ :

$$
\min _{\left(\boldsymbol{Q}_{12}, \boldsymbol{Q}_{p}\right) \in \mathcal{G}_{1}} \operatorname{tr}\left(\boldsymbol{Q}_{12}+\boldsymbol{Q}_{p}\right)
$$

where 


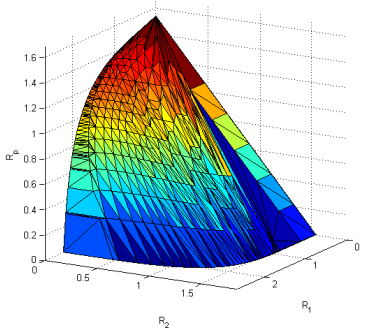

(a) Capacity region

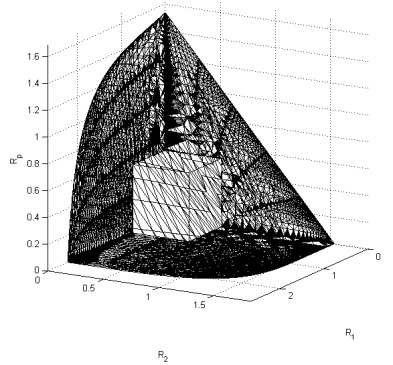

(b) Comparable TDMA approach
Fig. 3. Capacity region of the MISO Gaussian BBC with additional private message, with $N_{R}=2, N_{1}=N_{2}=1, h_{1}=\left[\begin{array}{ll}2 & 0.4\end{array}\right]^{T}, h_{2}=\left[\begin{array}{ll}0.2 & 1.2\end{array}\right]^{T}$ and $P=5$. Fig. 3(b) compares the capacity region with the achievable rate region of a comparable TDMA approach (white box) which realizes the same routing task in three orthogonal time slots.

$$
\mathcal{G}_{1}=\left\{\begin{array}{c}
\boldsymbol{Q}_{12} \succeq \mathbf{0}, \boldsymbol{Q}_{p} \succeq \mathbf{0} \\
\left(\boldsymbol{Q}_{12}, \boldsymbol{Q}_{p}\right) \mid \begin{array}{l}
\frac{\boldsymbol{h}_{1}^{T} \boldsymbol{Q}_{12} \boldsymbol{h}_{1}}{\boldsymbol{h}_{1}^{T} \boldsymbol{Q}_{p} \boldsymbol{h}_{1}+\sigma_{1}^{2}} \geq \alpha \gamma_{1} \\
\frac{1}{\sigma_{2}^{2}} \boldsymbol{h}_{2}^{T} \boldsymbol{Q}_{p} \boldsymbol{h}_{2} \geq \alpha \gamma_{p} \\
\frac{1}{\sigma_{2}^{2}} \boldsymbol{h}_{2}^{T}\left(\boldsymbol{Q}_{12}+\boldsymbol{Q}_{p}\right) \boldsymbol{h}_{2} \geq \alpha \gamma_{2}
\end{array}
\end{array}\right\}
$$

and where $\alpha$ is an auxiliary non-negative parameter and the triplet $\left(\gamma_{p}, \gamma_{1}, \gamma_{2}\right)$ can be interpreted as received SNIR "weights".

By rewriting the constraint set of $\mathcal{G}_{1}$ as a linear constraint set, we obtain a linear semi-definite optimization problem and hence convex. Yet, this still falls short of finding a point on the boundary of $\mathcal{R}\left(\boldsymbol{Q}_{12}, \boldsymbol{Q}_{p}\right)$.

Obviously all rates increase as the auxiliary parameter $\alpha$ increases. Thus we obtain the weighted rate sum optimal rate triple on the boundary of $\mathcal{R}\left(\boldsymbol{Q}_{12}, \boldsymbol{Q}_{p}\right)$ for fixed weights $\gamma_{p}, \gamma_{1}, \gamma_{2}$, by finding the maximum $\alpha$ such that the constraint set provides at least one feasible solution. Finally, running through all weight vectors with $\gamma_{p}+\gamma_{1}+\gamma_{2}=1$ yields all weighted rate sum optimal rate triples and characterizes the boundary of $\mathcal{R}\left(\boldsymbol{Q}_{12}, \boldsymbol{Q}_{p}\right)$.

A visual representation of the capacity region (10) is presented in Figure 3(a) and a comparison of this region with the TDMA approach in Figure 3(b).

\section{CONClusion}

Physical layer service integration deals with the efficient implementation of different services at the physical layer. In this paper we studied physical layer integration of additional private message in bidirectional relay networks which basically required the study of the discrete memoryless $\mathrm{BBC}$ and multi-antenna Gaussian BBC with additional private message. We established the entire capacity region which characterize the fundamental limits up to which rates bidirectional and private messages can be transmitted in bidirectional relay networks for each case. Finally, for the MISO scenario with multiple transmit antennas but single receive antenna, we found a way to convert the non-convex optimization problem into a convex one which allows to compute the weighted rate-sum optimal rate triples. This yields the boundary of the capacity region which is compared with the TDMA approach.

\section{REFERENCES}

[1] E. Biglieri, R. Calderbank, A. Constantinides, A. Goldsmith, A. Paulraj, and H. V. Poor, MIMO Wireless Communications. Cambridge University Press, 2007.

[2] T. J. Oechtering, C. Schnurr, I. Bjelaković, and H. Boche, "Broadcast Capacity Region of Two-Phase Bidirectional Relaying," IEEE Trans. Inf. Theory, vol. 54, no. 1, pp. 454-458, Jan. 2008.

[3] S. J. Kim, P. Mitran, and V. Tarokh, "Performance Bounds for Bidirectional Coded Cooperation Protocols," IEEE Trans. Inf. Theory, vol. 54, no. 11, pp. 5235-5241, Nov. 2008.

[4] G. Kramer and S. Shamai (Shitz), "Capacity for Classes of Broadcast Channels with Receiver Side Information," in Proc. IEEE Inf. Theory Workshop, Tahoe City, CA, USA, Sep. 2007, pp. 313-318.

[5] R. F. Wyrembelski, T. J. Oechtering, I. Bjelaković, C. Schnurr, and H. Boche, "Capacity of Gaussian MIMO Bidirectional Broadcast Channels," in Proc. IEEE Int. Symp. Inf. Theory, Toronto, Canada, Jul. 2008, pp. 584-588.

[6] T. J. Oechtering, R. F. Wyrembelski, and H. Boche, "Optimal Transmit Strategy for the 2x1 MISO Bidirectional Broadcast Channel," in Proc. IEEE Signal Process. Adv. Wireless Commun., Recife, Brazil, Jul. 2008, pp. 316-320.

[7] T. J. Oechtering, E. A. Jorswieck, R. F. Wyrembelski, and H. Boche, "On the Optimal Transmit Strategy for the MIMO Bidirectional Broadcast Channel," IEEE Trans. Commun., vol. 57, no. 12, pp. 3817-3826, Dec. 2009.

[8] T. J. Oechtering, H. T. Do, and M. Skoglund, "Achievable Rates for Embedded Bidirectional Relaying in a Cellular Downlink," in Proc. IEEE Int. Conf. Commun., Cape Town, South Africa, May 2010, pp. $1-5$.

[9] R. F. Wyrembelski and H. Boche, "Physical Layer Integration of Private, Common, and Confidential Messages in Bidirectional Relay Networks,' IEEE Trans. Wireless Commun., vol. 11, no. 9, pp. 3170-3179, Sep. 2012.

[10] A. Sezgin, A. S. Avestimehr, M. A. Khajehnejad, and B. Hassibi, "Divide-and-Conquer: Approaching the Capacity of the Two-Pair Bidirectional Gaussian Relay Network," IEEE Trans. Inf. Theory, vol. 58, no. 4, pp. 2434-2454, Apr. 2012.

[11] H. Degenhardt and A. Klein, "Filter Design with Optimized Numbers of Data Streams for Multi-Pair Two-Way Relaying Under Asymmetric Rate Requirements," in Proc. ITG Workshop Smart Antennas, Dresden, Germany, Mar. 2012, pp. 294-300.

[12] E. Yilmaz, R. Zakhour, D. Gesbert, and R. Knopp, "Multi-pair Two-way Relay Channel with Multiple Antenna Relay Station," in Proc. IEEE Int. Conf. Commun., Cape Town, South Africa, May 2010, pp. 1-5.

[13] F. Roemer and M. Haardt, "Tensor-Based Channel Estimation and Iterative Refinements for Two-Way Relaying With Multiple Antennas and Spatial Reuse," IEEE Trans. Signal Process., vol. 58, no. 11, pp. 5720-5735, Nov. 2010.

[14] T. M. Cover and J. A. Thomas, Elements of Information Theory, 2nd ed. Wiley \& Sons, 2006.

[15] A. El Gamal and Y.-H. Kim, Network Information Theory. Cambridge University Press, 2011.

[16] M. Salehi, "Cardinality Bounds on Auxiliary Variables in Multiple-User Theory via the Method of Ahlswede and Körner,' Technical Report, Aug. 1978.

[17] I. Csiszár and J. Körner, "Broadcast Channels with Confidential Messages," IEEE Trans. Inf. Theory, vol. 24, no. 3, pp. 339-348, May 1978.

[18] G. Kramer, "Teaching IT: An identity for the Gelfand-Pinsker converse," IEEE Inf. Theory Society Newsletter, vol. 61, no. 4, pp. 4-6, Dec. 2011.

[19] H. Weingarten, Y. Steinberg, and S. Shamai (Shitz), "The Capacity Region of the Gaussian Multiple-Input Multiple-Output Broadcast Channel," IEEE Trans. Inf. Theory, vol. 52, no. 9, pp. 3936-3964, Sep. 2006.

[20] _ , "On the Capacity Region of the Multi-Antenna Broadcast Channel with Common Messages," in Proc. IEEE Int. Symp. Inf. Theory, Seattle, WA, USA, Jul. 2006, pp. 2195-2199. 